\title{
Watching paint dry: Magnetic resonance imaging of soft condensed matter
}

\author{
Peter J. McDonald and Joseph L. Keddie \\ Department of Physics, University of Surrey, UK
}

Solid, crystalline matter is now remarkably well understood, $\mathcal{S}$ thanks mainly to the efforts of physicists during the last century. Building on this success, there has been an increasing trend among physicists in the past few decades to turn their attention to soft condensed matter - or "squidgy" stuff. Soft matter displays either viscous (liquid-like) or elastic (solid-like) behaviour, depending on the time scale of the measurement. Examples range from gelatine and pastes to liquid crystals and melted polymers. Physicists' interest in soft matter arises in part because it displays an intriguing universality in behaviour and can be described by "coarse-grained" models that ignore atomistic and chemical detail. A characteristic of soft matter is its tendency to arrange itself at hierarchical levels, such as the layering in liquid crystals and the ordering of colloidal particles into a cubic array. As such, the relevant length scales range between the molecular (nanometer) up to tens of micrometers.

Many types of soft matter, such as concentrated emulsions, are not stable under high vacuum and are perturbed by even light mechanical forces. Phases that are confined to small volumes can only be studied by techniques that do not disturb the confining phase. Soft matter is continuously undergoing thermal fluctuations, and so its structure is dynamic. Because of all of these characteristics, it is not feasible to probe soft matter by many analytical techniques. Non-invasive and fast techniques are required.

Natural substances, such as cells and tissues, can also be considered to be soft condensed matter. As aptly stated by William Burroughes, we humans are "soft machines." In 1973 two groups independently developed a technique to "look inside" these soft machines. Sir Peter Mansfield and colleagues at the University of Nottingham and Paul Lauterbur at the State University of New York in Stonybrook both announced that the resonance of magnetic nuclei could be exploited to non-invasively provide cross-sectional images in the technique known as magnetic resonance imaging (MRI).

MRI soon became the imaging modality of choice in medical research and diagnosis. It is now coming of age in the study of soft condensed matter, too. The stage through which all new microscopies go - that of taking "pretty pictures" as the primary objective - has truly passed. Now, enabled largely by physicists across Europe, scientists are starting to answer some questions of real import to the study of soft matter.

\section{Principles of MRI}

Magnetic resonance relies upon the fact that magnetic nuclei of atoms, such as the hydrogen proton, precess in a magnetic field at a frequency directly proportional to the field strength. The frequency, which is in the radio-frequency ( $\mathrm{r}-\mathrm{f}$ ) range, is detected via the current arising from the transient response of the nuclei to a resonant $\mathrm{r}-\mathrm{f}$ stimulus. This current is induced in a detector coil around the sample. Magnetic resonance imaging, as depicted in figure 1 , is achieved by superimposing on the sample a magnetic field gradient. The resonance frequency now encodes position. Fourier transform of the transient response yields an intensity versus frequency plot which directly correlates to a onedimensional density versus position profile. Imaging in multiple ( 2 and 3 ) spatial dimensions is achieved with multiple acquisitions under gradients in different directions followed by multi-dimensional Fourier transformation.

The lifetime of the transient decay (known as the spin-spin relaxation time, $T_{2}$ ), or equivalently the resonance linewidth $\left(1 / \pi T_{2}\right)$, as well as the relaxation time necessary to re-establish thermal equilibrium after excitation (known as the spin-lattice relaxation time, $T_{1}$ ) are exquisitely sensitive to motion at the molecular level. In a field gradient, they are sensitive to macroscopic motion such as flow. By carefully tailoring the sequences of $r-f$ pulse stimuli and the strength and sequencing of the magnetic field gradient applications, it is possible to sensitise the signal and thereby to map a wide variety of motion-sensitive parameters, including $T_{1}$ and $T_{2}$, as well as self-diffusion coefficients $D_{s}$ and flow velocities $\psi$.

It is this capability to non-invasively visualise the structure and dynamics of soft condensed matter that gives MRI considerable advantage over other microscopies and that more than compensates for its relatively poor best achievable resolution (a

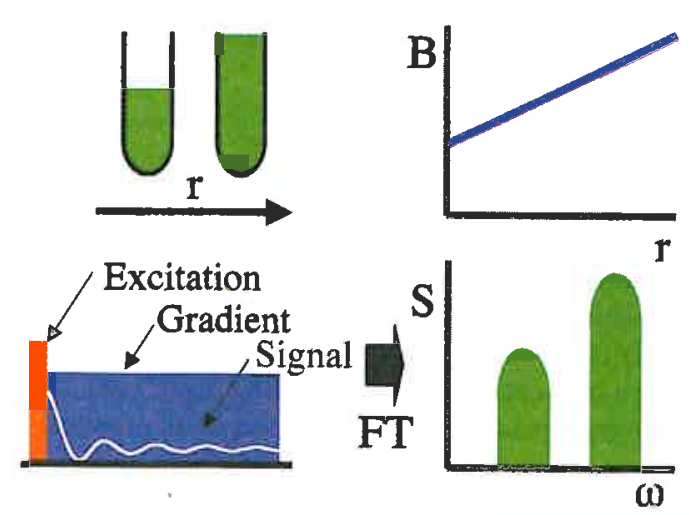

Eic. 1: The principle of magnetic resonance imaging: The Larmor equation states that the resonant frequency of magnetic nuclei, $\omega$, in a magnetic field is directly proportional to the field strength, $B$, according to $\omega=\gamma B$, where $\gamma$ is the nuclear magneto-gyric ratio. For spatial resolution, a switched gradient field $(g . r)$ is superimposed on a static field $\left(B_{\theta}\right)$ so that the resonant frequency $\omega=\gamma^{\prime}\left(B_{0}+g . r\right)$ encodes position, $r$ (top right). In this example, the sample consists of one full and one half-full test tube of water (top left).The hydrogen proton NMR signal is excited with an electro-magnetic radiofrequency excitation pulse and is observed as a transient response in a suitable receiver (bottom left). Fourier transformation of the response yields a frequency spectrum, $S(\omega)$ which has a direct one-to-one correspondence with the sample density profile (bottom right). 
few $\mu \mathrm{ms}$ ). Dependent on methodology, temporal resolution can be as good as $100 \mathrm{~ms}$, but for most of the studies reported here is more like 10 to 100 seconds.

Those exploiting the dependence of relaxation parameters, i.e. $T_{1}$ and $T_{2}$, and transport parameters, such as $D_{s}$ and $v$, on local environment to probe, with or without spatial localisation, the structure of soft matter are a very diverse group of researchers. At one extreme are researchers such as Brian Hills (UK) and Mike McCarthy (USA) who make detailed micro-structural studies of foodstuffs and their basic ingredients. Others, such as Lynn Gladden, Ken Packer (both in the UK) and Igor Koptyug (Russia) explore liquids in confined media and related transport phenomenon. Olle Söderman (Sweden) is not alone in making detailed studies of emulsions. Rainer Kimmich (Germany) has made detailed analyses of the motional spectrum of polymer chain in the melt and in confined geometries. The scope of much of this work is revealed in recent proceedings $[1,2]$. Few researchers, however, have specifically looked at colloidal films, such as paint, with MRI.

Aqueous colloids, in which sub- $\mu \mathrm{m}$ solid or liquid particles are dispersed in water, are ideal subjects for MRI. Here we review the application of MRI to the drying of layers of polymer colloids, or, less prosaically, to drying paint. We show that this proverbially tedious subject is in fact a rich source of physical interest and unsolved problems.

\section{Imaging of Aqueous Colloids}

In spite of the fact that aqueous colloids dry every day in thousands of guises - not least including spilt coffee [3]- this remains a problem of considerable theoretical interest and practical importance. MRI can offer unique insights as it can map the evolution of the water distribution in a drying layer as a function of time. By way of introduction, figure 2 summarises the key stages of the process by which polymer particles form a paint film.

Pioneering MR studies at the University of Surrey began with a study of convex layers of emulsions made from a viscous oil (alkyd) [4]. An emulsion is a finely-divided mixture of one liquid in another - in this case, alkyd droplets in water. Alkyd emulsions are being developed as a new, environmentally-friendly gloss paint. MRI reveals the fine detail of the transport of the alkyd and the water phases as the water evaporates. This work went on to consider much more generally the drying of aqueous dispersions.

Figure 3 shows cross-sectional images of the water content as a function of time after casting a dispersion of polymer particles on a glass substrate. The water can freely evaporate upwards. The images were obtained using a standard high field $(9.4 \mathrm{~T})$ super conducting NMR magnet equipped with standard switched current winding gradients for imaging. The images reveal how the drop dries laterally with the edges drying long before the central regions. Quantitatively describing this is not so simple. As the edges dry, so the particle concentration grows non-uniformly and this drives a lateral transport of water across the drop. Water flows from the central region, in which particles are dispersed in water, to the edge region, where they are packed closely together. The rate of water loss at the centre is thus more rapid than the average, and that at the edge is slower.

At what point in the process does drying from the edges first occur? Water is initially pinned to the edge of the layer because of strong capillary forces. Just as the capillary force draws water upward against the pull of gravity, so too can it prevent water from moving inward from the edges of a film. In this case, the tiny space between particles of radius $r$ in contact creates a capillary having a pressure that is proportional to $\sigma / r$, where $\sigma$ is the

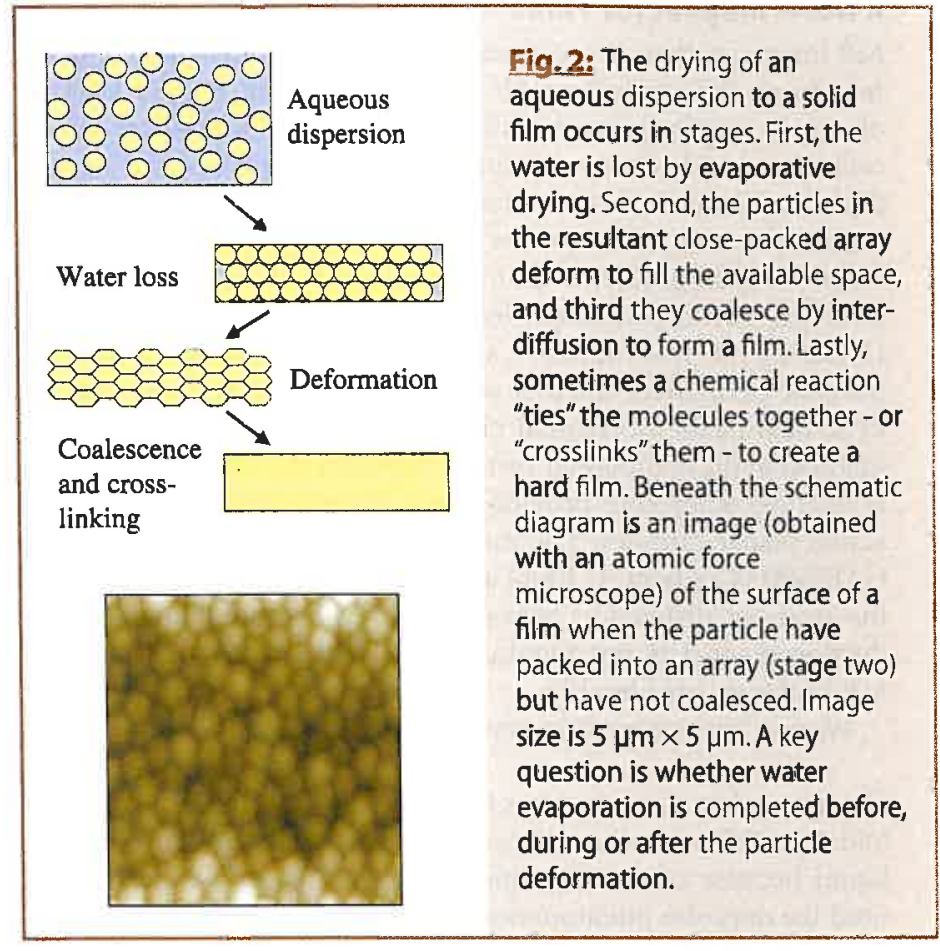

surface tension of water. At some instant, marking what is called the "open time", the capillary pressure is no longer strong enough to prevent the water from receding from the film edge.

Routh and Russel [5] at Princeton University have investigated this phenomenon theoretically in great detail. The critical parameters include particle size, film thickness, evaporation rate, surface tension, and viscosity. Uniform drying is favoured by larger particles, slower evaporation rates and thinner films. The analysis predicts a normalised time for drying of a film's edge as a function of reduced capillary pressure $p_{c}$. Using MRI data, such as that just presented, the Surrey group provided the first experimental test of Routh and Russel's predictions [6]. The data shown in figure 4, obtained from MR experiments, shows an encouraging agreement with the theory.

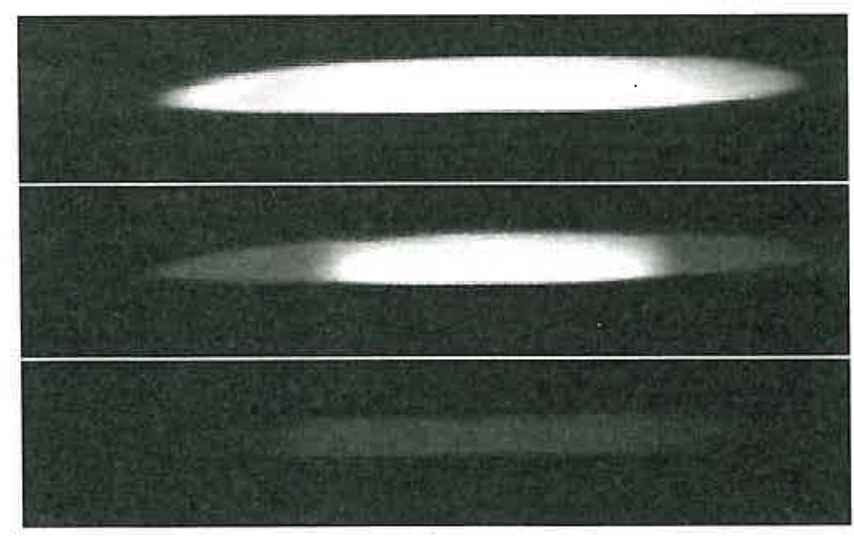

Fig. 3: Three images extracted from a series showing the drying of a latex film from the edges towards the centre [6]. From top to bottom are images of the initial wet film and after drying for three and six hours. The image intensity indicates the water content: the white regions contain particles still dispersed in water; the grey regions correspond to a close-packed array of particles with water flooding the interstices. The field of view is $22 \mathrm{~mm}$ wide by $2 \mathrm{~mm}$ deep. 


\section{A Novel Magnet for Films}

MR images, such as those already presented, do offer some information on the vertical profile of thick layers but thinner layers obviously require greater spatial resolution. At lower water concentrations and when accessing non-aqueous phases, the $T_{2}$ of the nuclei is much shorter. Studies of thin layers, low water concentrations and non-aqueous phases all require much greater magnetic field gradients than can normally be achieved using switched current-windings. To that end Paul Glover (now at the University of Nottingham) and colleagues designed a small portable permanent magnet with a uniform (magnitude) magnetic field in the horizontal plane and with a very strong field gradient in the orthogonal vertical direction [6]. This field profile is carefully tailored to provide best achievable spatial resolution across planar samples. The magnet (figure 5), which is dubbed GARField (Gradient At Right angles to Field), is ideally suited to the study of dispersion layers. Moreover, because a superconducting magnet is not required, GARField reduces the cost of MR profiling considerably.

We now cite contrasting examples of GARField's use.

Creaming. Just as cream rises to the top of whole fresh milk, colloidal particles in other dispersions likewise separate from the liquid because of density differences. The theory of creaming (and the opposite phenomenon of sedimentation) is well-developed but, because of the difficulty in non-invasively probing small particles in water, experimental work is still catching up. The use of NMR diffusometry to determine the size of emulsion droplets has long been well-established in a technique known as $q$-space microscopy. A more recent development is $q$-space microscopy coupled with imaging. In our application, the resultant MR profiles can determine how factors such as particle size and the viscosity of the water influence the creaming rate and the particle distribution.

Larger liquid droplets separate from a dispersion by creaming at a faster rate than smaller droplets. Naturally, therefore, larger droplets are found at the top of a cream layer. MRI studies of cream layers in simple oil-in-water emulsions confirm this expectation. The addition of water-soluble polymers to an emulsion can cause emulsion droplets to cluster together under the action of osmotic pressure resulting from depletion effects. MR analysis has found that these clusters - or flocs, as they are known - create cream layers that are much less stratified. The droplet size distribution is roughly constant throughout the layer.

Uniformity of drying. Most of us have touched the surface of a drying latex paint and noticed that its surface has formed a skin-like layer that covers a wetter region below. GARField is an ideal tool to probe the uniformity of drying in the direction normal to the surface of a paint layer.

The problem can be considered to be a competition between two effects, illustrated in figure 6. The particles diffuse by Brownian motion, described with a Stokes-Einstein diffusion coefficient $D$, and thereby will re-distribute themselves uniformly in a dispersion. When water evaporates at a rate $E$, however, particles become more crowded together near the surface as the water between them is removed. If the rate of re-distribution by diffusion is slower than the rate of particle

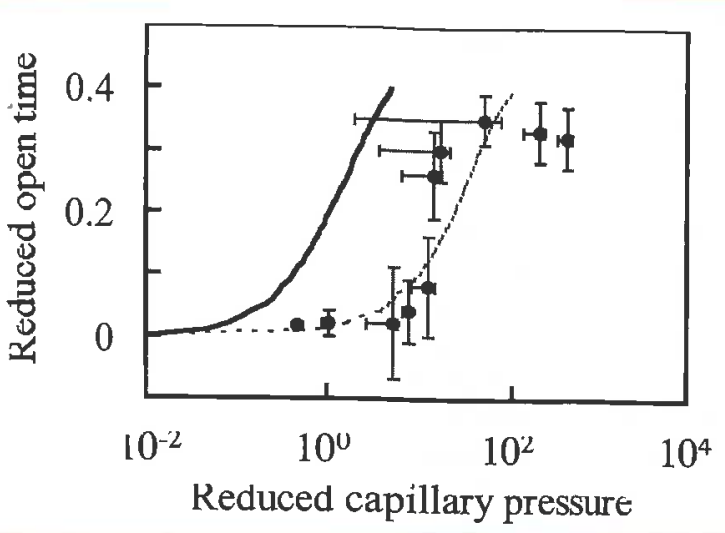

Fig.4: The solid line shows the reduced open time of a drying aqueous dispersion against reduced capillary pressure, as predicted by the model of Routh and Russel [5]. The open time is a measure of the time required for the water in the drop to begin to recede from the edges. The reduced capillary pressure depends on factors of geometry, viscosity, and surface energy. The data points are derived from MR images of 10 different drying films, spanning three orders of magnitude of reduced capillary pressure [6]. Although the data follow the broad shape of the simulation, the predicted capillary pressure where the open time rises steeply is too low. The dashed line is a re-evaluation based on less realistic geometric parameters.

crowding caused by water evaporation, then the water concentration will become non-uniform with less water near the surface. For a wet film with a thickness of $H$, Routh and Russel [7] propose a Peclet number (given as $H E / D$ ) to gauge the relative effects of diffusion and evaporation.

Recent GARField experiments have explored the correlation between the Peclet number and the distribution of water normal to the surface of a film. The predictions are supported by initial experiments. Additives that increase the viscosity of the aqueous phase and slow down diffusion favour non-uniform drying, whereas slow rates of evaporation lead to a uniform water distribution.

Particle deformation. The water loss in a packed bed of hard particles - such as sand - is not as simple as one might first expect. Air flows in to replace the water and creates a rough meniscus in a mechanism known as "viscous fingering". When drying soft particles, the problem is more complicated, because the particles are not static. The water phase can create a capillary pressure that "pushes down" on the particles and can sometimes deform them. Evaporation from a

Fig. 5: A wire-frame drawing of the GARField magnet, a small bench-top permanent magnet with shaped pole pieces which give a horizontal constant (magnitude) field in the horizontal plane and a strong in-built field gradient in the vertical direction (17 $\mathrm{T} \mathrm{m}^{-1}$ at $0.7 \mathrm{~T}$ ). It offers optimal resolution $(\approx 10 \mu \mathrm{m})$ for profiling softsolid layers and has revolutionised our ability to examine paint layers, among other systems [7]. The inset shows the magnet as realised by Resonance Instruments Ltd. The curved pole pieces of the magnet are seen in the centre of the box, which is $60 \mathrm{~cm}$ wide. 
